\title{
EL DERECHO POSITIVO O CONVENCIONAL, SU NATURALEZA: EL CARÁCTER CREATIVO (CONSTITUTIVO)Y DERIVADO DE LAS LEYES HUMANAS
}

Sebastián Contreras Aguirre

THE POSITIVE OR CONVENTIONAL LAW, ITS NATURE. THE CREATIVE AND DERIVED CHARACTER OF HUMAN LAWS

\section{RESUMO}

EL SIGUIENTE TRABAJO INTENTA PRESENTAR ALGUNOS CRITERIOS PARA RECONOCER UNA NORMA DE DERECHO POSITIVO. SI BIEN ES CIERTO QUE PARA EL IUSNATURALISMO CLÁSICO UNA MISMA NORMA CONTIENE ELEMENTOS NATURALES Y CONVENCIONALES, ES POSIBLE, CREO, DETERMINAR SI UNA DISPOSICIÓN CUALQUIER PERTENECE, STRICTO SENSU, AL DERECHO POSITIVO O AL DERECHO NATURAL. EN PARTICULAR, SE PROPONE QUE UNA REGLA DE JUSTICIA CONVENCIONAL MANTIENE UNA SITUACIÓN INTERMEDIA ENTRE LAS LLAMADAS NORMAS ÓNTICAS Y LOS PRINCIPIOS TERCIARIOS DE LA LEY NATURAL. AUnque COMPARTE ELEMENTOS CON ESOS TIPOS DE REGLAS DE JUSTICIA, NO PUEDE SER CLASIFICADA NI COMO DERECHO NATURAL TERCIARIO NI COMO UNA NORMA DE JUSTICIA QUE CREA EX NIHILO NUEVOS ESTADOS DE COSAS.

\section{PALAVRAS-CHAVE}

DERECHO POSITIVO; DERECHO NATURAL; NATURALEZA DE LAS

NORMAS DE DERECHO POSITIVO; IUSNATURALISMO CLÁSICO.

\section{ABSTRACT}

THIS PAPER ATTEMPTS TO PRESENT SOME CRITERIA FOR RECOGNIZING A RULE OF POSITIVE LAW. EVEN THOUGHIT IS TRUE THAT THE CLASSICAL IUSNATURALISM TEACHES THAT THE LEGAL NORMS CONTAINS NATURAL AND CONVENTIONAL ELEMENTS, IT IS POSSIBLE, I THINK, RECOGNIZE THAT A RULE BELONGS, STRICTLY SPEAKING, AT THE POSITIVE LAW OR NATURAL LAW. IN PARTICULAR, I PROPOSE THAT A RULE OF CONVENTIONAL JUSTICE IS IN AN INTERMEDIATE SITUATION BETWEEN ONTIC TERTIARY RULES AND PRINCIPLES OF NATURAL LAW.

\section{KEYWORDS}

Positive law; natural Just; nature of POSITIVE LAW; CLASSICAL IUSNATURALISM.

\section{Planteamiento}

Podemos decir que una norma pertenece a lo justo convencional cuando contiene algún elemento que no se deduce desde la ley natural, es decir, siempre que haya dispuesto un "nuevo" contenido de justicia, originariamente inexistente según el orden del derecho natural.

Esto no significa que el poder normativo de la autoridad entrañe el ejercicio de una libertad absoluta. La actividad inventiva de la autoridad es libre, pero dentro de los márgenes del derecho natural. Luego, su capacidad normativa no es completa, 
sino que más bien consiste en una libertad afectada. Por ende, a pesar de que las leyes humanas sean el resultado de las elecciones libres de la autoridad, si no existiera un principio objetivo que valide su obligatoriedad y justicia, esas disposiciones humanas no serían sino el resultado del abuso de poder. Este principio de legitimidad del derecho convencional se encuentra en la propia razón. Por eso se ha escrito que la justicia de las leyes escritas depende de su grado de conformidad o disconformidad con la norma de la razón, que es la ley de la naturaleza. ${ }^{1}$

Pese a esto, en su tarea de creación de las leyes positivas, el legislador debe no solo considerar la justicia de la ley natural; también debe considerar la contingencia de las condiciones de lugar y tiempo de su comunidad. Este proceder tanto desde los principios universales como desde los particulares es lo propio de la razón práctica, y, en particular, de la virtud de la prudencia. En su operación, y justamente por tratar acerca de lo singular, siempre existe un elemento de riesgo e inseguridad. ${ }^{2}$

Esto sucede porque, a pesar de que lo bueno moral en lo práctico sea, en principio, común a todas las clases de acciones, no lo es en lo particular, donde no existe el mismo nivel de seguridad que en los conocimientos universales, sino que al contrario: todo queda sometido a la contingencia de la praxis. Por tal motivo, las normas del derecho convencional no son simples explicitaciones de lo justo natural, sino el resultado de un proceso creativo del gobernante, que consiste en la ponderación de unos principios de justicia universales y unas condiciones particulares de tiempo y lugar. Es esto lo que explica que las leyes positivas sean un objeto cultural, en el sentido de una creación humana establecida libremente por las elecciones de la autoridad, elecciones que deben, sin embargo, someterse a las prescripciones de aquellas verdades morales objetivas que hemos llamado de ley o justicia natural.

\section{LAS DETERMINACIONES ENTRE DERECHO NATURAL TERCIARIO Y REGLAS ÓNTICAS}

La ley positiva, para serlo verdaderamente, debe guardar una cierta distancia con la ley natural. Esa distancia, en todo caso, no puede ser tal que entre lo justo positivo y lo justo natural no exista ninguna vinculación. Lo que proporciona fuerza obligatoria a las leyes humanas es, precisamente, su condición de normas derivadas del derecho natural. Por ende, distancia aquí no significa completa desvinculación entre normas positivas y derechos naturales.

Ahora bien, la correcta inteligencia de la naturaleza de las convenciones es posible, solamente, si consideramos que son, por una parte, normas derivadas de los principios naturales (en alguna medida, "conclusiones"3 particulares de esos principios), y, por otra parte, reglas de justicia "puestas" por la autoridad, en el sentido de “creadas” por el poder normativo del Estado. El problema, sin embargo, radica en que ambas condiciones se deben cumplir siempre para caracterizar a una norma como 
derecho convencional, porque, como ya ha enseñado Cicerón, aunque dispongan nuevos contenidos de justicia, las leyes humanas siempre derivan de las leyes naturales.

No basta, por tanto, con que esa regla de justicia sea concluida desde lo justo por naturaleza. Tampoco es suficiente que el legislador la haya definido ex nihilo, esto es, sin referencia a los principios morales. Entonces, antes de revisar la naturaleza de las leyes positivas, es preciso distinguir dos categorías de normas que, en nuestra opinión, permiten comprender la naturaleza de las convenciones: primero, las normas del derecho natural terciario (o principios terciarios de la ley natural); y, segundo, las llamadas reglas de justicia óntico-constitutivas. ${ }^{4}$

En cuanto al derecho natural de tercer orden, es necesario indicar que se forma por el conjunto de conclusiones que, remotamente, se derivan por modo de deducción desde los principios primarios de la ley natural. Lo que ocurre, escribe Tomás de Aquino, es que "todos los preceptos morales son de ley natural, aunque de diverso modo. Y así, hay unos que cualquiera, con su razón natural, entiende que se deben hacer o evitar". ${ }^{5}$ Hay otros, en cambio, que se conocen fácilmente, pero no a través de una aprehensión intelectual, sino por medio de la razón discursiva, que, avanzando en el conocimiento práctico, concluye unas verdades morales más particulares a partir de los llamados communissima. De esta condición son los principios del Decálogo, por ejemplo: "con la misma lógica que se sigue de que todo hombre es risible, el que Pedro es hombre, luego es risible; así se sigue correctamente de este principio: no hieras a nadie, que es un principio natural; matar es herir, luego, no es lícito matar a nadie". 6

Por último, hay otros principios "que se imponen después de la atenta consideración de los sabios, y estos son de ley natural, pero tales que necesitan de aquella disciplina con que los sabios instruyen a los rudos". ${ }^{7}$ En estos, "la fuerza de la consecuencia no es evidente sino muy probable, y así hace falta la doctrina de los grandes y sabios para que sean recibidas, como: no prestar para la usura, que se sigue de esto: no robarás, pero no tan claro que no pueda alguien dudar que por el beneficio mutuo puedan recibir pago". 8

Se plantea el siguiente problema:

cómo conocemos que algo es natural y que obliga si no es evidente para todos que es natural. A esto se responde que aquellas cosas que pertenecen al primer grado del derecho natural obligan a todos, porque esas cosas son muy patentes a todos por su propia naturaleza. De manera similar, las que pertenecen al segundo grado obligan a todos porque todos de modo evidente, por un conocimiento natural, conocen que ellas se deducen de los primeros principios de la naturaleza. ${ }^{9}$

En el caso de los principios terciarios, 
vale la siguiente proposición: que cuando existen algunas razones suficientemente claras de que algo sea de derecho natural, si por otra parte no existen razones que prueben que lo contrario no es contra el derecho natural, tal ha de sostenerse que es de derecho natural a pesar de que a los hombres corruptos y malos no les sea evidente. ${ }^{10}$

Por su parte, las reglas ónticas son normas que no hacen referencia a ninguna entidad preexistente, como sí ocurría con lo justo natural en el caso de los principios terciarios. No expresan lo que descriptivamente es algo, sino que crean una realidad ex nihilo: ${ }^{11}$ "antes de la regla el algo no existe, y solo mediante la regla ese algo existe”. ${ }^{12}$ Se trata, entonces, de disposiciones que asumen una función ónticofundacional en los sistemas normativos, ${ }^{13}$ y que deciden el espacio, el tiempo, los sujetos y las competencias de las realidades jurídicas que por ellas son creadas. ${ }^{14}$

Desde la perspectiva lingüística, esas reglas se expresan mediante el verbo ser, que en la norma tiene un carácter prescriptivo (o directivo) y no puramente predicativo (o descriptivo). ${ }^{15}$ Este tipo de reglas no son ni verdaderas ni falsas, porque no pueden contrastarse con una realidad previa que defina su contenido veritativo. Tampoco les afecta el requisito normativo de la coherencia. "Por el contrario, son las reglas ónticas las que crean el marco de coherencia respecto de las demás reglas, aunque es problemático que pueda hablarse incluso aquí de coherencia propiamente dicha." 16 Por lo tanto, se trata de normas que crean un ámbito óntico-práctico, esto es, una cierta realidad convencional.

Ejemplos de estas normas son: "la capital de Argentina es Buenos Aires", "en el escudo patrio habrá un cóndor y un huemul”. Tales normas crean el estado de cosas que mandan, como la capitalidad. Ahora bien, es evidente que estas normas no crean las ciudades-capitales en su sentido físico, "esto es, como conjunto de casas, calles, parques, etc. El concepto de 'capitalidad' es jurídico y no físico”. 17

Al comienzo de este apartado hemos indicado que las convenciones o leyes humanas se encuentran en una situación intermedia entre los principios terciarios de la ley natural y las reglas ónticas. Esto sucede porque: (i) con las normas del derecho natural terciario comparten el hecho de consistir en una forma de "conclusiones lejanas" de los communissima; y (ii) con las reglas óntico-constitutivas comparten el hecho de que se trata de leyes que crean contenidos de justicia nuevos, que originalmente no eran parte del sistema político.

No obstante, no son ni normas ónticas ni normas del derecho natural terciario. No son normas ónticas, porque, a pesar de que el derecho positivo pueda disponer contenidos de justicia inexistentes en la ley natural, se trata de un derecho "derivado": la normatividad de lo justo por naturaleza se halla contenida en sus disposiciones. Luego, acá no procede una forma de creatio ex nihilo como en el caso de las reglas constitutivas. 
Tampoco son formas de derecho natural terciario. Aun cuando, en sentido amplio, se pueda hablar de "conclusiones" para referirse a las normas del derecho positivo, lo cierto es que las normas del derecho humano no se obtienen deductivamente desde los principios primarios de la ley natural. No existe una forma de concatenación lógica que permita relacionar los communissima con las determinaciones, como sí la hay en las normas naturales de tercer grado. ${ }^{18}$

\section{EL PROBLEMA DE LA POTESTAD MARITAL Y LOS CRITERIOS} PARA RECONOCER UNA DETERMINACIÓN O NORMA POSITIVA

Para las doctrinas ilustradas, el derecho natural representa una realidad normativa ahistórica e intemporal. Los hechos quedan subsumidos en las normas al modo de fenómenos que no sufren cambio, de forma tal que el procedimiento jurídico se concibe a la manera de la geometría.

Tal ha sido la idea de Thomasius, por ejemplo, quien señala que "la moral está sujeta a demostración en la misma medida que las matemáticas". ${ }^{19}$ De la misma manera, escribe Spinoza: "consideraré las acciones y los apetitos humanos como si se tratara de líneas, de superficies y de cuerpos sólidos". ${ }^{20}$ Esto significa concebir al derecho como un teorema: 21 "hasta la más concreta de las reglas de derecho, será deducida del principio tomado apriorísticamente como postulado". 22

En nuestra opinión, el motivo por el cual los autores del derecho natural racionalista extienden el campo de la deducción a principios de justicia que son, según nos parece, convenciones o normas positivas, radica en la importancia que esos autores entregaban a la certeza matemática en los razonamientos jurídicos: ${ }^{23}$ era precisa esa forma de certeza, "pues de no existir, cualquiera podía interpretar el contrato o la ley a su antojo, con lo cual la obligación quedaría en una situación imposible: lo que uno cree que debe cumplir no coincidiría con lo que otro cree que se le debe cumplir". ${ }^{24}$

Más arriba hemos señalado que las leyes positivas no pueden ser consideradas, en esencia, como normas de derecho natural, ni siquiera como normas de derecho natural terciario. Esto sucede porque entre los principios morales y las leyes positivas no existe una vinculación geométrico-deductiva. Luego, cuando se defiende que una norma es de derecho natural, siendo, en estricto sentido, una norma positiva, lo que ha sucedido es que, como enseña Santo Tomás, se ha determinado con mezcla de error. ${ }^{25}$

Un ejemplo de determinación errónea se da, según nos parece, en el caso de la potestad marital. Como se sabe, el filósofo había enseñado que la mujer no era apta para la vida política. Esta se encontraba destinada, naturalmente, a la generación y la vida en la comunidad doméstica. ${ }^{26}$ Y la razón de esto era simple: por naturaleza, el hombre era mejor que la mujer. ${ }^{27}$

En este sentido, según observa Fernández Concha, 
aunque la unión de los esposos se funda en el amor, y aunque este los requiera e incline a procurar de común acuerdo su mutua felicidad y la de sus hijos, por la diversidad de talentos, de caracteres y de propensiones pueden surgir entre ellos, ya más, ya menos, ya mayores, ya menores, disensiones en lo tocante a la fijación del domicilio, a la elección de la profesión, a la administración de los bienes, a la institución de la prole, o a cualquier otro punto del régimen interno o externo de la sociedad conyugal. En ésta, por lo tanto, del propio modo que en cualquiera otra, para asegurar la conspiración de las voluntades y de las operaciones en orden al fin común, se requiere un poder dotado de la facultad de elegir, determinar e imponer los medios convenientes al expresado fin. ${ }^{28}$

Ahora bien,

todo el género humano ha creído que ese primado doméstico compete al varón y no a la mujer, pues así se ha practicado siempre y por doquier; el cual hecho es un indicio suficiente de que tal es el orden natural. Que en esto no hay engaño lo demuestran la fisiología y la psicología, las cuales encuentran, por lo general, una no disputable y no pequeña superioridad física e intelectual del hombre sobre la mujer. ${ }^{29}$

Por esta causa, como escribe Spinoza,

si atendemos a la experiencia, veremos que la condición de las mujeres procede de su debilidad natural. En ninguna parte ha sucedido que los hombres y mujeres reinen juntos [...] Pero si las mujeres fuesen por naturaleza iguales a los hombres, en fortaleza de ánimo e inteligencia, en lo que consiste principalmente el poder y, por tanto, el derecho de los humanos, las mujeres estarían al mismo nivel que los hombres. Entre los pueblos numerosos y diferentes del mundo, se hallarían algunos en los que los dos sexos detentarían juntos la autoridad política, y otros en los que gobernasen las mujeres a los hombres, educándoles de modo que su inteligencia no se desarrollase. Como no se han producido hechos semejantes en parte alguna, es lícito afirmar, sin duda, que las mujeres no gozan naturalmente de un derecho igual al de los hombres y que son, naturalmente, inferiores. ${ }^{30}$

Los dos autores citados piensan que la potestad marital es de derecho natural. También Tomás de Aquino llega a esa conclusión. Esto se debe, como hemos señalado, a la mezcla de error que se puede dar en el establecimiento de las normas positivas, en este caso particular, al hecho de que, históricamente, hubo una primacía del hombre 
sobre la mujer, que, sin embargo, no se debía al derecho natural, sino a la práctica o una forma de ignorancia invencible entre los distintos pueblos.

Nadie podría defender hoy que la Tierra es cuadrada o que las manchas lunares no existen, porque el cielo es un lugar inmaculado. Por largos años se pensó que así eran las cosas, e incluso se creía que "por naturaleza” esas eran las verdades de la ciencia. Pero la experiencia nos muestra que las culturas pueden estar equivocadas en sus intuiciones, como en el caso de los germanos, que, según expone Santo Tomás, afirmaban que el robo era lícito.

Como el lector se habrá dado cuenta, nuestra conclusión es que la primacía del esposo en la sociedad conyugal es solo materia de convención. No existe una supremacía "por naturaleza" del hombre sobre la mujer que justifique la consideración de esa norma como de derecho natural. Por esta causa, es patente que por derecho de naturaleza no es incompatible con las mujeres la potestad de legislar.

Evidentemente, si por derecho de la naturaleza, una mujer fuera incapaz de recibir esa potestad legislativa, sería esencialmente injusto e intrínsecamente un mal que la mujer sucediera en el trono por derecho hereditario, o que fuera elegida como reina y juez; ahora bien, esto es absurdo y contra la experiencia de muchos reinos católicos legalmente instituidos. ${ }^{31}$

Todo lo dicho nos enfrenta al problema de cómo reconocer una determinación o norma de derecho positivo. A continuación intentaremos presentar algunos criterios para resolver este inconveniente. Antes bien, es necesario indicar que se ha llegado a sostener que las normas que establecen la obligación de indemnizar sin culpa, ${ }^{32} \mathrm{o}$, incluso, las que disponen que se forma una comunidad entre el que realiza de buena fe una obra con materiales ajenos y el dueño de esos materiales, ${ }^{33}$ son todas normas de derecho natural.

Hay buenas razones para pensar esto. Algo de la justicia natural se encuentra presente en esas disposiciones, porque todas ellas han sido derivadas, de alguna manera, desde la ley natural. Sin embargo, esto no significa que puedan identificarse como normas puramente naturales; eso sería tanto como desconocer el papel de la adinventio humana en la creación de la ley y del derecho positivo. ${ }^{34}$

A pesar de que en los ejemplos citados sea posible reconocer la presencia de un fundamento natural para decidir en un sentido o en otro, lo cierto es que, ni la imposición de una indemnización por responsabilidad sin culpa, ni la formación de una comunidad entre el que realiza la obra y el dueño de los materiales, pueden ser consideradas como de derecho natural. ${ }^{35} \mathrm{Y}$ es que "el derecho natural no desciende a ese tipo de minucias". 36

No obstante, éste no es un tema sencillo. De ahí que hayamos decidido bosquejar algunas reglas para el reconocimiento de una convención o norma de derecho 
positivo-humano. ${ }^{37}$ Un primer criterio para reconocer una convención o norma positiva es la materia de la ley. A diferencia de las normas naturales, que son necesarias per se, las reglas del derecho humano son contingentes, en cuanto que tratan sobre materias indiferentes, esto es, sobre aquellas cosas que no están exigidas al hombre por su naturaleza, sino solo por la voluntad del gobernante. ${ }^{38}$

Tal ha sido la idea de Aristóteles. Según observa el filósofo de Estagira,

lo justo político se divide en natural y legal; es natural el que en todas partes tiene la misma fuerza, y no por parecerlo o no parecerlo; y es legal, el que en un principio en nada difiere que sea así o de otro modo pero que una vez establecido sí difiere; por ejemplo: que el rescate cueste una mina, o que haya que sacrificar una cabra y no dos ovejas. ${ }^{39}$

Esto nos muestra que mientras que se trate de acciones o conductas que no han sido ordenadas o prohibidas por el derecho natural, el legislador tiene completa capacidad para definir algo como justo o injusto, siempre que eso no sea contrario al bien de la comunidad política. Un ejemplo de esto lo vemos a propósito de las normas que regulan el tránsito. No es la ley natural la que define el sentido del tránsito. A lo sumo promueve su regulación al disponer el orden social.

Sentado lo anterior, la autoridad debe adoptar una posición clara sobre cuál de todas las alternativas disponibles para la regulación del tránsito es la que conviene mayormente en unas determinadas circunstancias de tiempo y lugar. Esto es fundamental, porque, dada la contingencia que envuelve a la vida humana, la decisión del legislador francés, por ejemplo, no tiene por qué ser idéntica a la de las autoridades inglesas. Y, de hecho, mientras que el legislador francés "comprobó que no hay una obligación categórica de circular por la izquierda, y optó por la circulación por la derecha; el legislador inglés [...] optó por la otra alternativa, derivándose ambas por delegación de la ley natural”.40

En este sentido, el derecho natural no determina el sistema de circulación perfecto y definitivo para cada sociedad, ni prefija ninguna alternativa como la única y categóricamente correcta. Por el contrario, y justamente porque se trata de una materia indiferente a la ley natural, "habrá varios sistemas que acarrearán distintos riesgos, ventajas, costes y beneficios, a menudo inconmensurables, que cabrán dentro de las exigencias del derecho natural. Por tanto, el legislador podrá actuar con un amplio margen de libertad creativa al elegir y hacer obligatorio un sistema en particular entre las distintas opciones razonables". ${ }^{41}$

Un segundo criterio para reconocer una norma de derecho positivo está en la aplicación de la siguiente regla: "la decisión contraria no causa injusticia". ${ }^{42}$ Es decir, el legislador no está obligado a tomar una decisión en un sentido determinado; solo debe tomar una decisión que excluya otras alternativas posibles. ${ }^{43} \mathrm{El}$ ejemplo de la regulación del tránsito nos puede ayudar con este asunto: porque se trata de una materia 
indiferente en la que se presentan distintas opciones compatibles con el derecho natural, la elección de "por la derecha" en vez de "por la izquierda" no supone una contravención de las normas de la recta razón. El legislador inglés bien podría haber adoptado, en justicia, una decisión diferente.

Una tercera característica de las determinaciones es su carácter cambiante. Esto es, además de tratar sobre materias indiferentes a la ley natural y de que el legislador no está obligado en una cierta dirección, es propio de las leyes del derecho positivo su naturaleza mudable: cuando cesa la utilidad de una determinación o el motivo por la que se promulgó, esta deja entonces de mirar al bien de la comunidad; por eso debe ser modificada o dejada sin efecto. Esto se ve claramente en las normas que regulan el catálogo y definición de los estupefacientes, o en aquellas que dicen relación con los regímenes previsional, laboral, sindical y de seguridad social: ${ }^{44}$ todas estas materias son cambiantes por definición, aunque en razón de su conveniencia o inconveniencia para el bien común. ${ }^{45}$

En cuarto lugar, y volviendo al ejemplo de la regulación del tránsito, la elección de "por la izquierda" en vez de "por la derecha” representa un acto de la voluntad del gobernante. Esto no quiere decir que las normas positivas o convencionales sean únicamente el resultado de la voluntad de la autoridad política (esto convertiría nuestra tesis en una forma de voluntarismo), sino que en el ámbito de cosas igualmente razonables para el cumplimiento de la ley natural, es un acto de la voluntad el que especifica qué es lo justo en tales condiciones de tiempo y lugar.

Pero se trata de un acto de la voluntad que no procede ex nihilo. El acto del entendimiento se encuentra presupuesto en el movimiento de la voluntad, porque "el objeto de la voluntad es el bien en cuanto entendido; y en tal sentido la inteligencia opera como fin respecto de la voluntad". 46 Según esto, el apetito no puede autodeterminarse en la identificación de lo bueno o de lo malo moral. Necesita estar guiado por la inteligencia. De ahí que se le haya caracterizado como una potencia ciega: ${ }^{47}$ en su obrar, debe seguir el juicio práctico de la razón.

\section{CONSIDERACIONES FINALES}

Para los pensadores clásicos, el derecho natural no es un sistema normativo contrapuesto al derecho positivo o simplemente legal. En realidad, tampoco es un sistema, porque no consiste en un ordenamiento jurídico cerrado sobre sí, donde las reglas particulares de justicia deben deducirse geométricamente desde los principios universales del derecho. Para la tradición clásica, el derecho natural es una parte del único ordenamiento de justicia que es el derecho de la comunidad, donde además de las normas de justicia natural "existen otras leyes que consideradas en sí mismas no hallan razón para su observancia”, pero que una vez consideradas todas las circunstancias particulares de esa comunidad "se hace conveniente que sean observadas". ${ }^{48}$ 
En el primer caso, se trata de preceptos indemostrables, que proceden del instinto de la naturaleza, ${ }^{49}$ y que dan cuenta de una justicia y equidad evidentes. ${ }^{50}$ En el segundo caso, al contrario, se trata de normas que dependen del parecer humano, y que, por esa razón, "no son ni universales ni inmutables, sino, a la inversa, mudables y limitadas en cuanto a su ámbito de validez temporal y territorial". 51

Esta distinción es de primera importancia. En la enseñanza clásica, los derechos naturales representan un verdadero límite a la actividad creativa del legislador; y la razón de esto es simple: el derecho natural es la causa ejemplar de la ley humana. De manera que la libertad creativa del gobernante se manifiesta en su incapacidad para obligar en conciencia sin el sostén de un mandato natural. ${ }^{52}$

A pesar de su carácter constitutivo, las normas positivas no son formas de creatio ex nihilo. Es indubitable que las leyes humanas crean nuevo derecho, pero no al punto de establecer contenidos de justicia completamente independientes del derecho natural. En tal forma delimitan la actividad legislativa del gobernante, que, sin referencia a esos preceptos de justicia naturales o universales, no se puede hablar de una justicia o rectitud en las leyes humanas. Por tanto, en la actividad normativa del gobernante, lo que define a una disposición como justa es la presencia de esas "leyes comunes" de las que hablan los filósofos griegos (que son los principios naturales) en el cuerpo de las normas positivas. Es esa presencia de lo justo natural lo que hace que una determinada acción o situación sea verdaderamente justa.

Ahora bien, que la ley natural delimite la actividad del gobernante no significa que las normas positivas sean constituidas por ella o que sus contenidos sean establecidos por lo justo natural. Lo que significa es que la ley humana pretende el mismo fin que la ley natural, ${ }^{53}$ aun cuando se haga cargo de materias que de suyo no pertenecían a lo justo por naturaleza. Esto origina que aunque una ley humana no sea propiamente constituida por la ley natural, ni su materia se derive de ésta, sí al menos, y solo en algún grado, su fuerza obligatoria, "por pretender el mismo fin y obligar a los súbditos, por razones distintas y por diferentes grados de exigencia, a intentar alcanzarlo”. 54

: ARTIGO APROVADO (02/12/2013) : RECEBIDO EM 14/05/2013 
2 Cf. Javier Hervada, "Reflexiones acerca de la prudencia jurídica y el derecho canónico". Revista Española de Derecho Canónico, n. 16 (p. 415-451), 1961, p. 420 ss.

3 Ahora bien, en las determinaciones, solamente podemos hablar de unas "conclusiones" en sentido amplio. Se trata, como dice Martin Rhonheimer, no de conclusiones de un discurso racional inventivo-demostrativo, sino conclusiones de aquel discurso, práctico de un modo inmediato y referido a lo concreto, de la prudencia (legisladora). Estas conclusiones surgen, no de una inventio de la razón práctica, sino de lo que Santo Tomás reconoce como adinventio. Se trata, en definitiva, de aquellas determinaciones concretas ad quae natura non primo inclinat; sed per rationis inquisitionem ea homines adinvenerunt, quasi utilia ad bene vivendum. Cf. Martin Rhonheimer, Ley natural y razón práctica. Una visión tomista de la autonomía moral. Pamplona: Eunsa, 2000, p. 398.

4 Una primera aproximación a este asunto ha sido expuesta en: Sebastián Contreras, "Derecho positivo y derecho natural. Una reflexión desde el iusnaturalismo sobre la necesidad y naturaleza de la determinación”. Kriterion, v. 127, 2013, p. 43-62.

5 Santo Tomás, Summa Theologiae, I-II, q. 100, a. 1

6 Bartolomé Carranza, De Justitia, q. 57, a. 2, fol. 178r.

7 Santo Tomás, Summa Theologiae, I-II, q. 100, a. 1. En este sentido, escriben los escolásticos: "aquellas cosas que son de derecho natural en primer grado son per se nota. Segunda: aquellas que son de derecho natural en segundo grado no son per se nota. En efecto, aquello de no matar, no robar, se sigue de esto: no hagas a los otros lo que no quieres que te hagan a ti. Pero son o evidentes o facilísimamente mostrables. Tercero aquellas cosas que son de derecho natural en tercer grado ni son per se nota ni tan evidentes que hace falta la doctrina de los mayores para aceptarlas" (Domingo de Soto, De Justitia, q. 57, a. 2).

8 Domingo de Soto, De Justitia, q. 57, a. 2.

9 Bartolomé Carranza, De Justitia, q. 57, a. 2, fol. 178r (traducción ligeramente modificada).

10 Bartolomé Carranza, De Justitia, q. 57, a. 2, fol. 178r.

11 Cf. Gregorio Robles, Las reglas del derecho y las reglas de los juegos. Ensayo de teoría analítica del derecho. México DF: Unam, 1988, p. 126.

12 Robles, Las reglas del derecho y las reglas de los juegos, p. 126.

13 Cf. Juan Omar Cofré, "Las reglas óntico-constitutivas, fundamentos de la persona y la dignidad humana". Revista de derecho, v. 15 n. 2 (p. 37-58), 2003, p. 40 ss.

14 Cf. Gregorio Robles, Comunicación, lenguaje y derecho. Algunas ideas básicas de la teoría comunicacional del derecho. Madrid: Real Academia de Ciencias Morales y Políticas, 2009, p. 89.

15 Cf. Gregorio Robles, El derecho como texto. Cuatro estudios de teoría comunicacional del derecho. Navarra: ThomsonCivitas, 2006, p. 43.

16 Robles, Las reglas del derecho y las reglas de los juegos, p. 127.

17 Gregorio Robles, Teoría del derecho. Fundamentos de teoría comunicacional del derecho. Madrid: Thomson Reuters, 2010, p. 228.

18 Es importante recordar que la determinación no implica una derivación teoremática de principios de justicia desde las leyes naturales. Si bien Santo Tomás ha comparado los preceptos de la ley natural con los principios de la geometría, esto no significa que la determinación de los communissima constituya una tarea geométrica o lógicodeductiva. Cf. Santo Tomás, In Sententiarum, II, d. 24, q. 2, a. 3.

19 Christian Thomasius, Fundamentos de derecho natural y de gentes. Madrid: Tecnos, 1994, p. 6. 
20 Baruch Spinoza, Ética, Buenos Aires: Aguilar, 1973, p. 161.

21 Cf. Carlos Ignacio Massini, Sobre el realismo jurídico. Buenos Aires: Abeledo-Perrot, 1978, p. 111.

22 Massini, Sobre el realismo jurídico, p. 111.

23 Cf. Hugo Grocio, Del derecho de presa. Del derecho de la guerra y de la paz. Madrid: Centro de Estudios Constitucionales, 1987, p. 7.

24 Fernando Quintana, Prudencia y justicia en la aplicación del derecho. Santiago: Editorial Jurídica de Chile, 2001, p. 24.

25 Cf. Santo Tomás, In Ethicorum, V, lect. 12, n. 1024.

26 Cf. Ana Marta González, Moral, razón y naturaleza. Una investigación sobre Tomás de Aquino. Pamplona: Eunsa, 2006, p. 359

27 Cf. Santo Tomás, In Politicorum, I, lect. 3, n. 36.

28 Rafael Fernández Concha, Filosofía del derecho o derecho natural. Madrid:Tipografía Católica, 1887-1888, v. 2, p. 147.

29 Fernández Concha, Filosofía del derecho o derecho natural, v. 2, p. 147.

30 Baruch Spinoza, Tratado teológico político. Tratado político. Madrid: Tecnos, 1966, p. 261-262.

31 Francisco de Araújo, De Lege, p. 142. Asimismo, escribe Araújo: "aunque es un inconveniente que una mujer sea elegida por los hombres para tener las riendas del poder en el sentido en el que Aristóteles en Ethica (VIII) dijo que es la corrupción de la ciudadanía el hecho de que mande una mujer, sin embargo, no es un inconveniente, ni es impropio lo siguiente: obtener el reino por derecho hereditario y suceder en el reino como heredad paterna, o ser elegida por Dios que no puede equivocarse".

32 Cf. Camilo Tale, "Derecho de daños y derecho natural”. In: Eduardo Soto Kloss y Sergio Raúl Castaño (eds.). El derecho natural en la realidad jurídica y social. Santiago: Universidad Santo Tomás, 2005, p. 595 ss. A este respecto es preciso decir lo siguiente: la tradición del derecho natural defiende la idea de que no hay responsabilidad por los daños no intencionales; luego, no cabe, para esta escuela de pensamiento, una indemnización por daños sin culpa en los términos defendidos por el profesor argentino Camilo Tale. Cf. Finnis, Natural Law and Natural Rights, p. 288.

33 Cf. Hugo Grotius, De iure belli ac pacis libri tres. Hildesheim: Olms, 2006, v. 2, p. 19.

34 Cf. Contreras, Derecho positivo y derecho natural, p. 55

35 Cf. Contreras, Derecho positivo y derecho natural, p. 55 ss.

36 Ugarte, Curso de filosofía del derecho, p. 489.

37 Una primera versión de estas reglas en: Sebastián Contreras, "Ley natural y determinación del derecho positivo”. Praxis Filosófica, n. 33, 2011, p. 207-226.

38 Cf. Juan de Santo Tomás, Cursus Theologicus, disp. IX, a. 6, n. 11

39 Aristóteles, Ethica Nichomaquea, 1134b18-1135a5.

40 Georges Kalinowski, El problema de la verdad en la moral y en el derecho. Buenos Aires: Eudeba, 1979, p. 152.

41 Robert P. George, Entre el derecho y la moral. Navarra: Aranzadi-Thomson, 2009, p. 41.

42 Esta regla no puede ser aplicada para el caso de las normas naturales, donde, precisamente, decidir en sentido contrario (o en contra de lo que "por naturaleza" ha sido definido como bueno) es causa de injusticia. 
43 Cf. José Tomás Alvarado, La determinación del derecho en Santo Tomás. Santiago: Pontificia Universidad Católica de Chile (tesis de licenciatura), 1993, p. 14.

44 Cf. Constitución Política de la República de Chile, a. 63 n. 4.

45 La variabilidad es un elemento de la esencia de las determinaciones, pero el cambio de las normas positivas es algo que debe decidirse prudencialmente. A este respecto: Cf. Santo Tomás, Summa Theologiae, I-II, q. 97, aa. 1 y 2.

46 Mirko Skarica, "La ley natural según la mente de Santo Tomás como principio de la ley positiva”. In: José Luis Widow, Miguel Ayuso y Álvaro Pezoa (eds.). Razón y tradición. Santiago: Globo Ediciones, 2011, v. 2, p. 105.

47 Cf. Domingo de Soto, De Iustitia et Iure, I, q. 1, a. 1.

48 Santo Tomás, In Sententiarum, III, d. 3, a. 3 (por ambas citas).

49 Cf. San Isidoro, Ethimologiarum, V, 4, 1.

50 Cf. Santo Tomás, Summa Theologiae, II-II, q. 57, a. 3.

51 Agustín Squella, “¿Por qué vuelve a hablarse de derecho natural?”. Revista Chilena de Derecho, v. 22 n. 1 (p. 78 $85), 1995$, p. 80 .

52 Cf. Giuseppe Graneris, La filosofía del derecho a través de su historia y de sus problemas. Santiago: Editorial Jurídica de Chile, 1979, p. 74.

53 Cf. Luis de León, De Legibus, q. 7, a. 3.

54 Juan Castillo, El pensamiento jurídico-político de Fray Luis de León en el Tratado “De Legibus”. Burgos: Universidad de Burgos, 1998 (tesis de doctorado), p. 402.

\title{
Sebastián Contreras Aguirre
}

\author{
Álvaro del Portillo 12455 \\ Las Condes \\ scalauandes.cl
}

DOCTOR EN FILOSOF́́a

Profesor-InVEstigador de LA Facultad de Derecho de LA Universidad de los Andes, Chile 
